\title{
Effects of ultrasound-guided suprascapular nerve pulsed radiofrequency on chronic shoulder pain
}

\author{
Tolga Ergönenç ${ }^{1}$, Serbülent Gökhan Beyaz ${ }^{2}$
}

${ }^{1}$ Sakarya University Education and Research Hospital, Department of Anesthesiology and Reanimation, ${ }^{2}$ Sakarya University, Faculty of Medicine, Department of Anesthesiology and Algology, Sakarya, Turkey

\begin{abstract}
Aim: Pulsed radiofrequency (PRF) therapy has become increasingly popular in the treatment of chronic shoulder pain due to its long duration of action and non-destructive method. The aim of the study was to reveal the effects of PRF therapy of the suprascapular nerve (SSN) under ultrasound guidance (UG) in patients with chronic shoulder pain on both shoulder pain and function. Material and methods: This study included 74 patients diagnosed with at least one of the following: adhesive capsulitis, rotator cuff syndrome and impingement syndrome of shoulder. The PRF therapy of the SSN under UG was performed in those patients with a reduction of $50 \%$ or more Visual Analog Scale (VAS) score and those that reported healing in the active range of motion (AROM) in the diagnostic SSN block. The resting, motion and sleeping shoulder pain assessments of the patients were done with VAS score. The shoulder joint function was assessed with the Shoulder Pain and Disability Index (SPADI) questionnaire and the AROM of the joint was measured using a goniometer. Results: In 70 of the 74 patients a $50 \%$ or more reduction was found in the VAS score with diagnostic SSN block. After the PRF therapy of the SSN, the 15 th day, 1st month, 3rd month, and 6th month follow-up VAS averages, SPADI averages and the flexion, internal rotation, external rotation, and abduction values were statistically significantly lower than the baseline values $(\mathrm{p}<0.05)$. Conclusion: This study is the largest series in the literature evaluating the efficacy of PRF therapy of the SSN under UG and has shown that pain can be controlled quickly, for a long period of time, using ultrasound guided PRF therapy of the SSN in chronic shoulder pain.

Keywords: impingement syndrome; chronic shoulder pain; suprascapular nerve; pulsed radiofrequency
\end{abstract}

\section{Introduction}

Shoulder pain is the second most common musculoskeletal disease in adults, and it often becomes chronic $[1,2]$ due to treatment difficulties. Rotator cuff disease, shoulder impingement syndrome, adhesive capsulitis, tendinitis and degenerative disease may cause chronic shoulder pain [3], which can negatively affect upper extremity joint function and life quality by limiting the

Received 20.05.2018 Accepted 10.10.2018

Med Ultrason

2018, Vol. 20, No 4, 461-466

Corresponding author: Tolga Ergönenç

Sakarya University Education and Research

Hospital, Department of Anesthesiology and

Reanimation, Sakarya, Turkey

E-mail: tolgaergonenc@gmail.com

Phone: +90 5324859840

Fax: +90 2642553292 range of motion of the joint. The treatment of shoulder pain requires an all-around approach, including noninvasive methods (nonsteroidal anti-inflammatory drugs, physical therapy and rehabilitation), minimally invasive methods (intraarticular steroid injections and suprascapular nerve procedures) and surgical procedures [4-6].

The suprascapular nerve (SSN) provides $70 \%$ of the sensory innervation of the shoulder joint; therefore, SSN procedures (selective nerve blocks, steroid injections and radiofrequency applications) have been reported to be effective in the treatment of acute and chronic shoulder pain [7-9]. Although rapid pain control can be provided with a SSN block, its short duration of action limits its use in treatment [8]. Longer pain control can be achieved with a neurolysis or neurectomy, but these methods may cause irreversible paralysis in the supraspinatus and infraspinatus muscles. Pulsed radiofrequency (PRF) therapy has become increasingly popular in the treatment of chronic shoulder pain due to its long duration of action 
and the lack of any damage to the targeted and surrounding tissue, thereby reducing the risk of neural damage and neuritis [10].

In this study, we aimed to reveal the effects of PRF therapy of the SSN under ultrasound guidance (UG) in patients with chronic shoulder pain on both shoulder pain and function.

\section{Materials and methods}

\section{Patient selection}

This cross-sectional observational study was conducted after approval by the University Ethical Committee and written informed consent was obtained from all patients included in study. Patients over 18 years-old diagnosed with at least one of the following: adhesive capsulitis, rotator cuff syndrome or impingement syndrome of shoulder were included. Additionally, the patients had to have minimum Visual Analog Scale (VAS) score 4 and minimum Shoulder Pain and Disability Index (SPADI) score 60 despite a 3 months nonsteroidal anti-inflammatory oral analgesics and physical therapy (initial passive range of motion, followed by advanced strengthening exercise). Patients with chronic pain due to other diseases, with surgery on the same shoulder, allergy history from local anesthesia, abnormal coagulation tests, cardiac pacemakers, neuropathic pain associated with cervical disc disorder, malignancy, stroke or complications of diabetes were excluded.

The diagnoses were established after clinical history, physical examination and magnetic resonance imaging (MRI) evaluation. Physical examination of the shoulder included inspection, palpation, evaluation of range of motion (flexion, internal rotation, external rotation, abduction) and shoulder provocative tests (Neer's sign, Yergason test, Hawkins test, Apley scratch test, Drop-arm test, Spurling's test, "Clunk" sign). The MRI examinations were interpreted by radiologists and other physical examination and measurements were performed by an experienced anaesthesiologist and an orthopedic surgeon.

\section{Assessment of pain and functional status}

Before and after the SSN block and PRF therapy of the SSN, the resting, motion and sleeping pain assessments were completed using a VAS. The shoulder joint function was assessed using the SPADI questionnaire and the active range of motion (AROM) of the joint was measured using a goniometer.

\section{Diagnostic suprascapular nerve block technique}

Under operating room conditions, a peripheral venous catheter was applied to each patient while the electrocardiogram (ECG), noninvasive blood pressure and peripheral oxygen saturation were being monitored. The diagnostic block of the SSN under UG was applied by a nerve stimulator while the patient was in a sitting position. Following the appropriate skin disinfection, with UG (M5 Color Diagnostic Ultrasound System; Mindray, Shenzhen, China), a 22 G $80 \mathrm{~mm}$ needle (Stimuplex; B. Braun Melsungen AG, Melsungen, Germany) with a nerve stimulator (Stimuplex HNS 11; B. Braun Melsungen AG, Melsungen, Germany) was pushed forward towards the floor of the supraspinous fossa using an in-plane approach (fig 1). A nerve stimulator was set to deliver $0.4 \mathrm{~mA}$ current impulses of $0.1 \mathrm{~ms}$ duration at a frequency of $2 \mathrm{~Hz}$. The needle was inserted caudally into a sagittal plane until the muscle twitched (external rotation and/or abduction in the shoulder) were observed in synchrony with the stimulation. Then, $4 \mathrm{~mL}$ of $0.25 \%$ bupivacaine was released, after confirming that blood was not drawn by intermittent aspiration. The patients received no analgesics after the procedure.

\section{Pulsed radiofrequency application technique}

The PRF therapy of the SSN was performed in those patients with a reduction of $50 \%$ or more VAS score in the diagnostic block of the SSN. The PRF therapy of the SSN was administered under operating room conditions while the patient was monitored in a sitting position with UG. An ultrasound-guided 22 G 5-mm active-tip 100-mm radiofrequency needle (SC-K; Top Neuropole, Tokyo, Japan) was pushed forward to the floor of the supraspinous fossa using an in-plane approach. Sensorial stimulation was given at $50 \mathrm{~Hz}$ for 1 ms with a wide pulse. The PRF treatment was applied for 3 cycles at 42 ${ }^{\circ} \mathrm{C}, 45 \mathrm{~V}, 2 \mathrm{~Hz}$, and $20 \mathrm{~ms}$, with a wide wave for 120 $\mathrm{s}$ (NeuroTherm, Middleton, MA, USA). This was after obtaining maximal $0.4 \mathrm{~V}$ paresthesia in the area of the

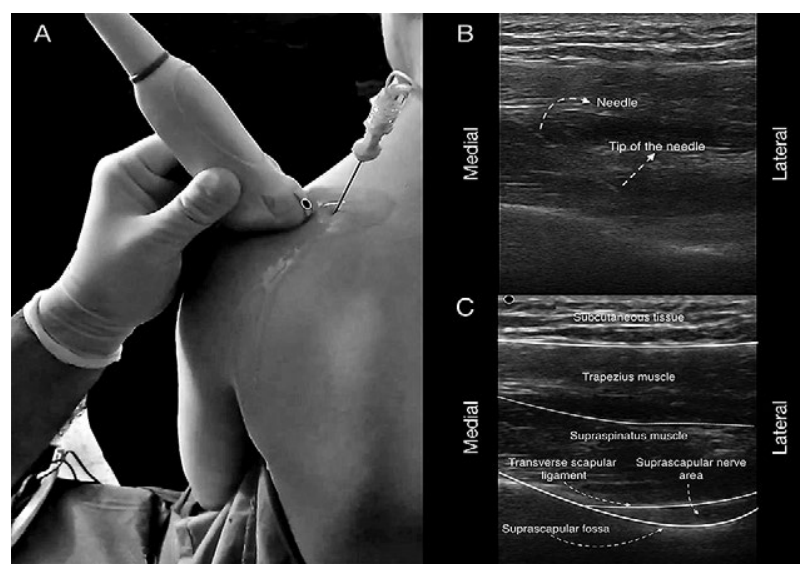

Fig 1. Ultrasonographic image of the suprascapular nerve on the floor of the supraspinous fossa. A: Positioning of the transducer and needle; B: Suprascapular fossa sonoanatomy and needle; C: Description of suprascapular fossa sonoanatomy 
suprascapular nerve innervation of the shoulder and after monitoring the shoulder abduction and/or external rotation response to the motor stimulus applied at $2 \mathrm{~Hz}$ for $1 \mathrm{~ms}$ and at least 1 volt. SPADI and AROM assessments were conducted postoperatively on the 15 th day, 1st month, 3rd month and 6th month follow-ups.

\section{Statistical Analysis}

In the data analysis, SPSS for Mac version 23.0 was used. The values were given as the mean \pm standard deviation $(\mathrm{SD})$ in the statistical analysis. The normality of distribution of the initial data was assessed using the Kolmogorov-Smirnov test. The demographic variables were compared using a t-test or chi-squared test for the continuous and categorical variables, respectively. The difference between the repeated measurements in the group was assessed using the Repeated Measures-ANOVA (RM-ANOVA). A p level of $<0.05$ was accepted for the statistical significance.

\section{Results}

In 70 of the 74 patients a $50 \%$ or more reduction was found in the VAS score during the diagnostic block of the SSN (94.59\%). The four patients without proper response to the diagnostic block were excluded. The demographic
Table I. Demographic characteristics of the patients

\begin{tabular}{ll}
\hline & $\mathbf{n}=\mathbf{7 0}$ \\
\hline Age (years) & $50.97 \pm 10.57$ \\
Sex (Male/Female) & $32 / 38$ \\
Side (Right/Left) & $36 / 34$ \\
Medical diagnosis & \\
$\quad$ Adhesive capsulitis & $17(24.29)$ \\
Rotator cuff syndrome & $22(31.43)$ \\
Shoulder impingement syndrome & $31(44.29)$ \\
\hline Data are given by mean + standard deviation and percentiles.
\end{tabular}

data of the 70 patients are detailed in table I. The average resting, movement, and sleep baseline VAS value of the 70 patients who underwent PRF therapy of the SSN was $4.65 \pm 0.85$. After the PRF therapy of the SSN, the 15 th day, 1st month, 3rd month, and 6th month follow-up VAS averages were statistically significantly lower than the baseline values $(p<0.05$, Table II).

The baseline SPADI average of the patients was 64.95 \pm 8.21 . After the PRF therapy of the SSN, the 15 th day, 1st month, 3rd month, and 6th month follow-up SPADI averages of the patients were statistically significantly lower than the baseline values $(p<0.05$, Table III).

Table II. Visual Analog Scale (VAS) values before and after pulsed radiofrequency therapy

\begin{tabular}{lllllll}
\hline VAS & Basal & $\mathbf{1 5}^{\text {th }}$ day & $\mathbf{1}^{\text {st }}$ month & 3 $^{\text {rd }}$ month & $\mathbf{6}^{\text {th }}$ month & p \\
\hline Resting & $1.49 \pm 0.79^{\dagger}$ & $1.29 \pm 0.76^{* *}$ & $1.03 \pm 0.78$ & $0.79 \pm 0.72^{\dagger}$ & $0.73 \pm 0.70$ & 0.48 \\
Movement & $6.44 \pm 1.20$ & $2.80 \pm 1.21$ & $2.20 \pm 1.31$ & $1.77 \pm 1.17^{\dagger}$ & $1.80 \pm 1.12$ & 0.07 \\
Sleep & $6.03 \pm 1.57$ & $2.41 \pm 1.57$ & $1.47 \pm 1.44^{\dagger}$ & $0.86 \pm 0.82^{\dagger}$ & $1.00 \pm 1.02$ & 0.12 \\
Mean score & $4.65 \pm 0.85$ & $2.17 \pm 0.82$ & $1.57 \pm 0.73$ & $1.14 \pm 0.49^{\dagger}$ & $1.18 \pm 0.53$ & $0.00^{*}$ \\
\hline
\end{tabular}

*Differences compared with basal value $(\mathrm{p}<0.05)$; **There were no differences compared with basal value $(\mathrm{p}>0.05)$; $†$ There were no differences compared with 6rd month ( $\mathrm{p}>0.05)$.

Table III. The Shoulder Pain and Disability Index score values before and after pulsed radiofrequency therapy

\begin{tabular}{lllllll}
\hline SPADI & Basal & $\mathbf{1 5}^{\text {th }}$ day & $\mathbf{1}^{\text {st }}$ month & $3^{\text {rd }}$ month & $\mathbf{6}^{\text {th }}$ month & p \\
Pain & $66.70 \pm 11.72$ & $44.77 \pm 12.92^{\dagger}$ & $42.99 \pm 13.66^{\dagger}$ & $41.83 \pm 14.26$ & $44.17 \pm 13.94$ & 0.28 \\
Disability & $63.20 \pm 12.05$ & $39.77 \pm 13.80$ & $38.73 \pm 13.88$ & $35.23 \pm 14.53$ & $34.60 \pm 14.59$ & $0.00^{*}$ \\
Mean score & $64.95 \pm 8.21$ & $42.27 \pm 9.62$ & $40.86 \pm 10.09$ & $38.53 \pm 10.63$ & $39.39 \pm 10.64$ & $0.00^{*}$ \\
\hline
\end{tabular}

SPADI: Shoulder Pain and Disability Index; ${ }^{*}$ Differences compared with basal value $(\mathrm{p}<0.05)$; $\dagger$ There were no differences compared with 6rd month $(\mathrm{p}>0.05)$.

Table IV. The active range of motion values before and after pulsed radiofrequency therapy

\begin{tabular}{lllllll}
\hline AROM & Basal & $\mathbf{1 5}^{\text {th }}$ day & $\mathbf{1}^{\text {st }}$ month & $\mathbf{3}^{\text {rd }}$ month & $\mathbf{6}^{\text {th }}$ month & p \\
\hline Flexion & $152.69 \pm 4.67$ & $170.94 \pm 5.82$ & $172.87 \pm 6.11^{\dagger \dagger}$ & $173.63 \pm 6.59$ & $166.47 \pm 7.07$ & 0.12 \\
Internal rotation & $60.44 \pm 4.46$ & $69.37 \pm 3.16$ & $71.07 \pm 2.89$ & $80.21 \pm 1.48$ & $75.17 \pm 5.95$ & $0.00^{*}$ \\
External rotation & $65.99 \pm 7.08$ & $70.64 \pm 7.18$ & $74.33 \pm 7.46^{\dagger}$ & $76.29 \pm 8.39$ & $74.29 \pm 8.73$ & 0.18 \\
Abduction & $97.10 \pm 15.19$ & $140.44 \pm 14.37$ & $155.06 \pm 12.47$ & $161.27 \pm 11.98$ & $159.37 \pm 11.76$ & $0.00^{*}$ \\
\hline
\end{tabular}

AROM: Active range of motion’*Differences compared with basal value $(\mathrm{p}<0.05)$; ${ }^{\dagger}$ There were no differences compared with $6^{\text {rd }}$ month $(p>0.05) ;{ }^{\dagger}$ There were no differences compared with $3^{\text {rd }}$ month $(p>0.05)$. 
Regarding the basal AROM averages of the patients after the PRF therapy of the SSN, at the 15th day, 1st month, 3rd month, and 6th month follow-ups, the average flexion, internal rotation, external rotation, and abduction values were statistically significantly higher than the baseline values ( $p<0.05$, Table IV).

No early and late complications were observed in any patient.

\section{Discussions}

In this study we determined that applying PRF therapy of the SSN in 3 cycles at $120 \mathrm{~s}$ each to patients with chronic shoulder pain (due to adhesive capsulitis, rotator cuff syndrome, or shoulder impingement syndrome) is effective for reducing shoulder pain, improving shoulder joint movement, and improving comfort. These positive effects lasted at least 6 months.

Chronic shoulder pain is caused most often by the structures in the shoulder $[2,11]$, and shoulder impingement syndrome is the most common role in its etiology. Medical treatment for shoulder pain is often difficult and unsuccessful. Interventional procedures maybe used in patients who do not benefit from conservative treatment [12]. After unsuccessful conservative treatment, it should be decided whether surgery is necessary to resolve the underlying causes of the complaints.

The SSN provide sensorial innervation of the posterior capsule of the glenohumeral joint, as well as the acromioclavicular joint, subacromial bursa and coracoclavicular ligament [13]. Therefore, the SSN is the focal point of many interventional procedures, including the SSN block, which is an effective method for controlling chronic shoulder pain $[8,14-16]$. However, the short duration of pain control provided by an SSN block limits its treatment uses [8].

The PRF therapy of the SSN has been increasingly used in the treatment of chronic shoulder pain due to its longer duration of pain control and the fact that it is nondestructive [10]. Unlike conventional radiofrequency, PRF does not cause any damage to the targeted and surrounding tissue, thereby reducing the risk of neural damage and neuritis [17-19]. Jang et al [20] observed that with a 240-pulse 50-V PRF therapy of the SSN applied to 11 patients with chronic persistent shoulder pain, at least 9 months of recovery was achieved in 10 of the patients. Lüleci et al [21] reported that they provided 6 months of pain control in 45 (78.9\%) of 57 patients using PRF treatment of the SSN, which they performed for $480 \mathrm{~s}$. In our study, we found that the $360 \mathrm{~s}$ ultrasound guidance PRF relieved pain, improved joint movement, and improved life quality for at least 6 months.
The PRF treatment of the SSN was performed by Jang et al [20] via C-arm fluoroscopy, while Lüleci et al [21] performed it blindly according to the anatomical landmarks. We performed the PRF therapy of the SSN under UG, due to its safety in experienced hands, thus reducing the complications (pneumothorax and intravascular injection), not including the radiation. In contrast to the other radiological imaging techniques, there was a higher success rate and a shorter treatment time [22].

There are a total of three publications in the literature, two of which were case reports, on PRF treatment of the SSN application via UG. Huang et al [23] described two patients diagnosed with frozen shoulders and Kim et al [24] described one patient with calcific tendinitis. Both of these reports described successful pain control for approximately 6 months with the PRF treatment of the SSN under UG. However, the first and only UG PRF treatment of the SSN study in the literature was conducted by Wu et al [25]. They reported that SSN PRF applied to 21 adhesive capsulitis patients, 12 weeks before physiotherapy, was more effective in improving joint function and pain control than in those patients treated with only physiotherapy for 12 weeks. Our research has provided the largest series in the literature on ultrasound-guided PRF therapy of the SSN with at least 6 months of recovery and pain control (similar to the time reported by $\mathrm{Wu}$ et al [25]).

In 32 disease cases in which the short-term and longterm efficacy of PRF on SSN were assessed by Keskinboraet al [10], decreased shoulder pain and increased shoulder joint movement at the 3rd week and 6th month were reported. In our study, we observed decreases in the VAS and SPADI values and increases in the AROM throughout the follow-up period, with short and longterm results compatible with those from Keskinbora et al study [10]. The remaining pain control at the 6th month follow-up after the PRF therapy of the SSN is important, since it indicates that PRF has a long-acting effect on nociceptive pain.

Gurbet et al [26] reported that they applied PRF on SSN twice for 120 seconds each to 8 patients with shoulder pain who had no response to medical treatment or physiotherapy. It provided an improvement in both pain control and range of motion for 3 months. Although there is insufficient data in the literature suggesting that applying $360 \mathrm{~s}$ of PRF therapy of the SSN may provide a longer duration of pain control comparing with $240 \mathrm{~s}$, it has been shown that the antiallodynic activity increases with an increased duration of PRF from 2 minutes to 6 minutes [27]. We applied PRF on SSN 3 times for $120 \mathrm{~s}$. 
Physiotherapy has an important role in the treatment of shoulder pain, but physiotherapy related pain can reduce patient compliance. Wu et al [25] reported that PRF therapy of the SSN treatments before physiotherapy improved the patient's compliance and tolerance. We found that at the 15th day after PRF therapy of the SSN, there was a significant decrease in the VAS score and increase in the AROM values, with healed shoulder joint function being provided. Physiotherapy can begin earlier thanks to PRF therapy of the SSN, which provides rapid pain control. A painless physiotherapy process can increase patient compliance and prevent chronic pain; nevertheless, there is insufficient evidence in the literature to recommend PRF therapy of the SSN routinely before physiotherapy.

There are several limitations of our study. Applying an invasive pseudo interventional procedure was an ethical problem to the control group. Therefore, the lack of a control group was a major limitation of our study. Second, the passive range of motion was not assessed in our study. In addition, although psychological factors may contribute to perception of pain they were not evaluated in this study.

\section{Conclusion}

Our study has shown that pain can be controlled quickly, for a long period of time, using ultrasoundguided PRF therapy of the SSN in chronic shoulder pain. There was significant recovery in the AROM in addition to this pain relief, along with a significant improvement in shoulder joint function. In chronic shoulder pain, PRF therapy of the SSN under UG can be applied safely as a nondestructive method, and that it can be repeated, if necessary. In order to verify our results additional large series, long-running, randomized controlled trials need to be conducted.

\section{Conflict of interest: none}

\section{References}

1. Picavet HS, Schouten JS. Musculoskeletal pain in the Netherlands: prevalences, consequences and risk groups, the DMC(3)-study. Pain 2003;102:167-178.

2. Herin F, Vézina M, Thaon I, Soulat J, Paris C; ESTEV group. Predictors of chronic shoulder pain after 5 years in a working population. Pain 2012;153:2253-2259.

3. Neer CS 2nd. Anterior acromioplasty for the chronic impingement syndrome in the shoulder. J Bone Joint Surg Am 2005;87:1399.
4. Liliang PC, Lu K, Liang CL, Tsai YD, Hsieh CH, Chen HJ. Pulsed radiofrequency lesioning of the suprascapular nerve for chronic shoulder pain: A preliminary report. Pain Med 2009;10:70-75.

5. Eyigor C, Eyigor S, Korkmaz OK, Uyar M. Intra-articular corticosteroid injections versus pulsed radiofrequency in painful shoulder: a prospective, randomized, single-blinded study. Clin J Pain 2010;26:386-392.

6. Gado K, Emery P. Modified suprascapular nerve block with bupivacaine alone effectively controls chronic shoulder pain in patients with rheumatoid arthritis. Ann Rheum Dis 1993;52:215-218.

7. Chansoria M, Das G, Mathankar N, Chandar D, Vyas N, Upadhyay S. A preliminary study of a novel technique of suprascapular nerve block in treating chronic shoulder pain. Indian J Pain 2015;29:91-95.

8. Shanahan EM, Ahern M, Smith M, Wetherall M, Bresnihan B, Fitzgerald O. Suprascapular nerve block (using bupivacaine and methylprednisolone acetate) in chronic shoulder pain. Ann Rheum Dis 2003;62:400-406.

9. Gofeld M, Restrepo-Garces CE, Theodore BR, Faclier G. Pulsed Radiofrequency of Suprascapular Nerve for Chronic Shoulder Pain: A Randomized Double-Blind Active Placebo-Controlled Study. Pain Pract 2013;13:96-103.

10. Keskinbora K, Aydinli I. Long-term results of suprascapular pulsed radiofrequency in chronic shoulder pain. Agri 2009;21:16-21.

11. Ainsworth R, Lewis JS. Exercise therapy for the conservative management of full thickness tears of the rotator cuff: a systematic review. Br J Sports Med 2007;41:200-210.

12. Schultz JS. Clinical evaluation of the shoulder. Phys Med Rehabil Clin N Am 2004;15:351-371.

13. Aszmann OC, Dellon AL, Birely BT, McFarland EG. Innervation of the human shoulder joint and its implications for surgery. Clin Orthop Relat Res 1996;(330):202207.

14. Vorster W, Lange CP, Briët RJ, et al. The sensory branch distribution of the suprascapular nerve: An anatomic study. J Shoulder Elb Surg 2008;17:500-502.

15. Vecchio PC, Adebajo AO, Hazleman BL. Suprascapular nerve block for persistent rotator cuff lesions. J Rheumatol 1993;20:453-455.

16. Wu Z, Wei LX, Li J, et al. Percutaneous treatment of non-contained lumbar disc herniation by injection of oxygen-ozone combined with collagenase. Eur J Radiol 2009;72:499-504.

17. Hagiwara S, Iwasaka H, Takeshima N, Noguchi T. Mechanisms of analgesic action of pulsed radiofrequency on adjuvant-induced pain in the rat: Roles of descending adrenergic and serotonergic systems. Eur J Pain 2009;13:249252.

18. Cahana A, Van Zundert J, Macrea L, Van Kleef M, Sluijter M. Pulsed radiofrequency: current clinical and biological literature available. Pain Med 2006;7:411-423.

19. Fukui S, Nitta K, Iwashita N, Tomie H, Nosaka S, Rohof O. Intradiscal pulsed radiofrequency for chronic lumbar discogenic low back pain: a one year prospective out- 
come study using discoblock for diagnosis. Pain Physician 2013;16:E435-E442.

20. Jang JS, Choi HJ, Kang SH, Yang JS, Lee JJ, Hwang SM. Effect of pulsed radiofrequency neuromodulation on clinical improvements in the patients of chronic intractable shoulder pain. J Korean Neurosurg Soc 2013;54:507-510.

21. Luleci N, Ozdemir U, Dere K, Toman H, Luleci E, Irban A. Evaluation of patients' response to pulsed radiofrequency treatment applied to the suprascapular nerve in patients with chronic shoulder pain. J Back Musculoskelet Rehabil 2011;24:189-194.

22. Lee HJ, Lim KB, Kim DY, Lee KT. Randomized Controlled Trial for Efficacy of Intra-Articular Injection for Adhesive Capsulitis: Ultrasonography-Guided Versus Blind Technique. Arch Phys Med Rehabil 2009;90:1997-2002.

23. Huang CC, Tsao SL, Cheng CY, Hsin MT, Chen CM. Treating frozen shoulder with ultrasound-guided pulsed mode radiofrequency lesioning of the suprascapular nerve: Two cases. Pain Med 2010;11:1837-1840.

24. Kim JS, Nahm FS, Choi EJ, Lee PB, Lee GY. Pulsed radiofrequency lesioning of the axillary and suprascapular nerve in calcific tendinitis. Korean J Pain 2012;25:60-64.

25. Wu YT, Ho CW, Chen YL, Li TY, Lee KC, Chen LC. Ultrasound-Guided Pulsed Radiofrequency Stimulation of the Suprascapular Nerve for Adhesive Capsulitis. Anesth Analg 2014;119:686-692.

26. Gurbet A, Türker G, Bozkurt M, Keskin E, Uçkunkaya N, Sahin S. Efficacy of pulsed mode radiofrequency lesioning of the suprascapular nerve in chronic shoulder pain secondary to rotator cuff rupture. Agri 2005; 17:48-52.

27. Tanaka N, Yamaga M, Tateyama S, Uno T, Tsuneyoshi I, Takasaki M. The effect of pulsed radiofrequency current on mechanical allodynia induced with resiniferatoxin in rats. 2010;111:784-790. 